\title{
MODULAR CALABI-YAU THREEFOLDS OF LEVEL EIGHT
}

\author{
SŁAWOMIR CYNK AND CHRISTIAN MEYER
}

\section{The $L$-Series of Rigid Calabi-Yau threefolds}

If $\tilde{X}$ is a Calabi-Yau threefold defined over $\mathbb{Q}$, and $p$ is a good prime (i.e., a prime such that the reduction of $\tilde{X} \bmod p$ is nonsingular) then the map

$$
\operatorname{Frob}_{p}^{*}: H_{\text {êt }}^{i}\left(\tilde{X}, \mathbb{Q}_{l}\right) \longrightarrow H_{\text {êt }}^{i}\left(\tilde{X}, \mathbb{Q}_{l}\right)
$$

on $l$-adic cohomology induced by the geometric Frobenius morphism gives rise to $l$-adic Galois representations

$$
\rho_{l, i}: \operatorname{Gal}(\overline{\mathbb{Q}} / \mathbb{Q}) \longrightarrow \mathrm{GL}_{b^{i}}\left(\mathbb{Q}_{l}\right) .
$$

If a Calabi-Yau threefold $\tilde{X}$ is rigid (i.e., $h^{1,2}(\tilde{X})=0$ or equivalently $\left.b^{2}(\tilde{X})=2\right)$ then $\tilde{X}$ is expected to be modular, i.e., the $L$-series of the (semi-simplification of the) two-dimensional Galois representation $\rho_{l, 3}$ associated with the middle cohomology $H_{\text {êt }}^{3}\left(\tilde{X}, \mathbb{Q}_{l}\right)$ equals the $L$-series of a cusp form $f$ of weight 4 for $\Gamma_{0}(N)$. The precise conjecture has been formulated by Saito and Yui in [10]. For details and examples the reader is referred to [14] or [6].

There are many examples of pairs of Calabi-Yau threefolds with an isomorphism between some pieces of their middle étale cohomologies and the appropriate Galois representations. In particular, if we can attach modular forms to these pieces then these modular forms will be the same. If on the other hand we detect the same modular forms in the middle étale cohomologies of two Calabi-Yau threefolds then this should have a geometrical reason:

Conjecture 1.1. (The Tate conjecture, as formulated in [14, Conj. 5.8]) If two isomorphic two-dimensional Galois representations $\rho_{1}, \rho_{2}$ occur in the étale cohomology of varieties $X_{1}, X_{2}$ defined over $\mathbb{Q}$, then there should be a correspondence between the two varieties (i.e., an

2000 Mathematics Subject Classification. 14G10, 14J32.

Key words and phrases. Calabi-Yau, double coverings, modular forms, Tate conjecture.

Partially supported by DFG Schwerpunktprogramm 1094 (Globale Methoden in der komplexen Geometrie) and KBN grant no. 1 P03A 00828. 
algebraic cycle on the product of the two varieties) defined over $\mathbb{Q}$, which induces an isomorphism between $\rho_{1}$ and $\rho_{2}$.

Following 4] we will call two Calabi-Yau threefolds defined over $\mathbb{Q}$ relatives if the same (weight four) modular form occurs in their $L$-series. Finding a correspondence between two relatives is a highly non-trivial task. It can be induced by a birational map defined over $\mathbb{Q}$ or more generally by a finite map between the two threefolds but this does not have to be the case. If a correspondence is induced by a birational map then by a result of Batyrev ([1]) the two Calabi-Yau threefolds must have the same Betti (and Hodge) numbers.

In this note we will deal with Calabi-Yau modular threefolds associated with the unique normalized weight four newform for $\Gamma_{0}(8)$. It can be written as a product

$$
f(q)=\eta(2 \tau)^{4} \eta(4 \tau)^{4}
$$

where $\eta(\tau)=q^{\frac{1}{24}} \prod_{n \in \mathbb{N}}\left(1-q^{n}\right), q=e^{2 \pi i \tau}$, is the Dedekind eta function. Some of the first Fourier coefficients $a_{p}$ of $f(q)=\sum_{n=1}^{\infty} a_{n} q^{n}$ are:

\begin{tabular}{|r|r|r|r|r|r|r|r|r|r|r|r|}
\hline$p$ & 2 & 3 & 5 & 7 & 11 & 13 & 17 & 19 & 23 & 29 & 73 \\
\hline$a_{p}$ & 0 & -4 & -2 & 24 & -44 & 22 & 50 & 44 & -56 & 198 & 154 \\
\hline
\end{tabular}

Note that by twisting with Legendre symbols we obtain newforms of different levels, for example 16, 64, 72, 144, 200, 392, 400. If these newforms occur in the $L$-series of some Calabi-Yau threefolds then we expect correspondences between them which are defined over some finite extension of $\mathbb{Q}$.

We will verify the Tate conjecture for rigid level 8 Calabi-Yau threefolds. More precisely we will give explicit correspondences between most known examples. Elliptic fibrations play a special role in the construction of correspondences, among them two modular curves from the Beauville list. This is not surprising as the Galois representation associated to the weight four level 8 newform is constructed using the self-fiber product of the modular curves $X_{\Gamma}$, for $\Gamma=\Gamma_{1}(4) \cap \Gamma(2)$ or $\Gamma=\Gamma_{0}(8) \cap \Gamma_{1}(4)$ (cf. 3]).

\section{List of LEVEL 8 Rigid CALABI-YAU threefoldS}

In this section we list all known examples of rigid Calabi-Yau threefolds associated with the weight four level 8 newform $f$. We are using the notation $T_{h^{1,1}}$. Examples with the same Hodge numbers are listed separately (with different superscripts) if no birational correspondence between them is known. 
2.1. Type $T_{70}, h^{1,1}=70$. Consider the affine threefold $Z$ given by the equation

$$
x+\frac{1}{x}+y+\frac{1}{y}+z+\frac{1}{z}+t+\frac{1}{t}=0 .
$$

It has been studied in detail in [9]. It fibres via projection to $\mathbb{P}^{1}(x)$ into so called Fermi surfaces and is therefore called Fermi threefold.

There are various birational Calabi-Yau models of $Z$. The invariants are

$$
\chi=140, \quad h^{1,1}=70, \quad h^{2,1}=0 .
$$

Verrill ([13] ) considers $Z$ as a double cover of the toric variety associated with the root lattice $A_{1}^{3}$.

Changing the signs of $z$ and $t$ we can rewrite the equation for $Z$ as

$$
\frac{(x+y)(x y+1)}{x y}=\frac{(z+t)(z t+1)}{z t} .
$$

This way we obtain a birational equivalence between $Z$ and the selffiber product of the Beauville elliptic surface $Y_{\Gamma}$ with $\Gamma=\Gamma_{0}(8) \cap \Gamma_{1}(4)$. In the literature a small resolution of the self-fiber product is denoted by $W_{0}(8)$. The singular fibers in this case are of the following types:

$$
\begin{array}{llll}
I_{8} & I_{2} & I_{1} & I_{1} \\
I_{8} & I_{2} & I_{1} & I_{1}
\end{array}
$$

Fiber products of elliptic fibrations were first studied in detail by Schoen (cf. 11]). Examples and correspondences with other CalabiYau threefolds are investigated in sections 4 and 5 .

By homogenizing the equation for $Z$ we find a birational model as a quintic in $\mathbb{P}^{4}$ defined by the equation

$$
w^{2}(x y z+x y t+x z t+y z t)=x y z t(x+y+z+t) .
$$

This quintic is birationally equivalent with the double covering of $\mathbb{P}^{3}$ branched along the union of five planes and a Cayley cubic which can be given by the equation

$$
u^{2}=x y z t(x+y+z+t)(x y z+x y t+x z t+y z t) .
$$

2.2. Type $T_{70}^{1}, h^{1,1}=70$. Let $X$ be the double covering of $\mathbb{P}^{3}$ branched along the octic surface

$$
x y z t(x-y)(y-z)(z-t)(t-x)=0 .
$$

It occurs as arrangement no. 2 in [2] and as arrangement no. 1 in [6]. Resolving the singularities of $X$ we obtain a Calabi-Yau threefold with invariants

$$
\chi=140, \quad h^{1,1}=70, \quad h^{2,1}=0 .
$$


2.3. Type $T_{50}, h^{1,1}=50$. Let $V_{1}$ be the double covering of $\mathbb{P}^{3}$ branched along the octic surface

$$
x y z t(x+y)(y+z)(x-y-z-t)(x+y-z+t)=0 .
$$

It occurs as arrangement no. 29 in [2] and as arrangement no. 32 in 6]. Resolving the singularities of $V_{1}$ we obtain a Calabi-Yau threefold with invariants

$$
\chi=100, \quad h^{1,1}=50, \quad h^{2,1}=0 .
$$

Let $V_{2}$ be the double covering of $\mathbb{P}^{3}$ branched along the octic surface

$$
x y z t(x+y)(x-y+z)(x-y-t)(x+y-z-t)=0 .
$$

It occurs as arrangement no. 44 in [2] and as arrangement no. 69 in [6]. Resolving the singularities of $V_{2}$ we obtain a Calabi-Yau threefold with the same invariants as those of $V_{1}$. In fact a birational correspondence between $V_{1}$ and $V_{2}$ is exhibited in section 3

2.4. Type $T_{46}, h^{1,1}=46$. Let $X$ be the double covering of $\mathbb{P}^{3}$ branched along the octic surface

$$
x y z t(x+y)(x-y+z)(y-z-t)(x+z-t)=0 .
$$

It occurs as arrangement no. 62 in 2 and as arrangement no. 93 in 6]. Resolving the singularities of $X$ we obtain a Calabi-Yau threefold with invariants

$$
\chi=92, \quad h^{1,1}=46, \quad h^{2,1}=0 .
$$

2.5. Type $T_{44}, h^{1,1}=44$. Let $X$ be the double covering of $\mathbb{P}^{3}$ branched along the octic surface

$(x-t)(x+t)(y-t)(y+t)(z-t)(z+t)(x+y+z+t)(x+y+z-t)=0$.

It occurs as arrangement no. 87 in 2 2 and as arrangement no. 238 in 6. Resolving the singularities of $X$ we obtain a Calabi-Yau threefold with invariants

$$
\chi=88, \quad h^{1,1}=44, \quad h^{2,1}=0 .
$$

The projective coordinate change

$$
(x: y: z: t) \mapsto\left(-\frac{y+z}{2}+t:-x-\frac{y+z}{2}:-\frac{y+z}{2}-t: \frac{y-z}{2}\right)
$$

transforms the branch locus into the octic surface given by

$$
(x-y)(x+y)(y-z)(y+z)(z-t)(z+t)(t-x)(t+x)=0 .
$$

The projective coordinate change

$$
(x: y: z: t) \mapsto(x-t: y-z: y+z: x+t)
$$


transforms this equation into

$x y z t(x+y+z-t)(x+y-z+t)(x-y+z+t)(-x+y+z+t)=0$.

2.6. Type $T_{40}, h^{1,1}=40$. Let $X$ be the double covering of $\mathbb{P}^{3}$ branched along the octic surface

$$
x y z t(x+y+z+t)(x+y-z-t)(y-z+t)(x+z-t)=0 .
$$

It occurs as arrangement no. 241 in [6]. Resolving the singularities of $X$ we obtain a Calabi-Yau threefold with invariants

$$
\chi=80, \quad h^{1,1}=40, \quad h^{2,1}=0 .
$$

2.7. Type $T_{40}^{1}, h^{1,1}=40$. Consider a crepant resolution of a fiber product of two elliptic fibrations with the following types of singular fibers:

$$
\begin{array}{llll}
I_{2} & I_{2} & I_{4} & I_{4} \\
I_{2} & I_{2} & I_{4} & I_{4}
\end{array}
$$

It is the self-fiber product of the Beauville elliptic surface $Y_{\Gamma}$ with $\Gamma=\Gamma_{1}(4) \cap \Gamma(2)$. In the literature the resolution is denoted by $W_{1}(4)$. It is a Calabi-Yau threefold with invariants

$$
\chi=80, \quad h^{1,1}=40, \quad h^{2,1}=0 .
$$

2.8. Type $T_{40}^{2}, h^{1,1}=40$. Consider a crepant resolution of a fiber product of two elliptic fibrations with the following types of singular fibers:

$$
\begin{array}{cccc}
I_{2} & I_{2} & I_{4} & I_{4} \\
I_{2} & I_{2} & D_{4}^{*} & I_{2}
\end{array}
$$

It is a Calabi-Yau threefold with invariants

$$
\chi=80, \quad h^{1,1}=40, \quad h^{2,1}=0 .
$$

2.9. Type $T_{40}^{3}, h^{1,1}=40$. Consider the complete intersection $X$ of four quadrics in $\mathbb{P}^{7}$ given by the equations

$$
\begin{aligned}
& u_{1}^{2}=x^{2}-y^{2}, \\
& u_{2}^{2}=y^{2}-z^{2}, \\
& u_{3}^{2}=z^{2}-t^{2}, \\
& u_{4}^{2}=t^{2}-x^{2} .
\end{aligned}
$$

Resolving the singularities of $X$ we obtain a Calabi-Yau threefold $\tilde{X}$ with invariants

$$
\chi=80, \quad h^{1,1}=40, \quad h^{2,1}=0 .
$$

This is explained in detail in section 3 . 
2.10. Type $T_{36}, h^{1,1}=36$. Consider a crepant resolution of a fiber product of two elliptic fibrations with the following types of singular fibers:

$$
\begin{array}{ccc}
I_{2} & D_{6}^{*} & I_{2} \\
D_{6}^{*} & I_{2} & I_{2}
\end{array}
$$

It is a Calabi-Yau threefold with invariants

$$
\chi=72, \quad h^{1,1}=36, \quad h^{2,1}=0 .
$$

2.11. Type $T_{32}, h^{1,1}=32$. Consider the complete intersection $X$ of four quadrics in $\mathbb{P}^{7}$ given by the equations

$$
\begin{aligned}
& 2 y_{0}^{2}=+x_{0}^{2}-x_{1}^{2}-x_{2}^{2}-x_{3}^{2}, \\
& 2 y_{1}^{2}=-x_{0}^{2}+x_{1}^{2}-x_{2}^{2}-x_{3}^{2}, \\
& 2 y_{2}^{2}=-x_{0}^{2}-x_{1}^{2}+x_{2}^{2}-x_{3}^{2}, \\
& 2 y_{3}^{2}=-x_{0}^{2}-x_{1}^{2}-x_{2}^{2}+x_{3}^{2} .
\end{aligned}
$$

It has 64 ordinary nodes as only singularities. There exist projective small resolutions of all the nodes. The invariants of a small resolution $\tilde{X}$ of $X$ are

$$
\chi=64, \quad h^{1,1}=32, \quad h^{2,1}=0 .
$$

The threefold $\tilde{X}$ has been studied in detail by Nygaard and van Geemen in 8 .

2.12. Type $T_{32}^{1}, h^{1,1}=32$. Consider a crepant resolution of a fiber product of two elliptic fibrations with the following types of singular fibers:

$$
\begin{array}{cccc}
I_{4} & I_{4} & I_{2} & I_{2} \\
I_{2} & I_{2} & D_{4}^{*} & I_{2}
\end{array}
$$

It is a Calabi-Yau threefold with invariants

$$
\chi=64, \quad h^{1,1}=32, \quad h^{2,1}=0 .
$$

2.13. Type $T_{32}^{2}, h^{1,1}=32$. Consider a crepant resolution of a fiber product of two elliptic fibrations with the following types of singular fibers:

$$
\begin{array}{llll}
I_{2} & I_{2} & I_{4} & I_{4} \\
I_{4} & I_{4} & I_{2} & I_{2}
\end{array}
$$

It is a Calabi-Yau threefold with invariants

$$
\chi=64, \quad h^{1,1}=32, \quad h^{2,1}=0 .
$$


2.14. Type $T_{28}, h^{1,1}=28$. Let $X$ be the double covering of $\mathbb{P}^{3}$ branched along the octic surface

$\left(x^{2}+y^{2}+z^{2}-t^{2}\right)\left(x^{2}+y^{2}-z^{2}+t^{2}\right)\left(x^{2}-y^{2}+z^{2}+t^{2}\right)\left(-x^{2}+y^{2}+z^{2}+t^{2}\right)=0$.

Resolving the singularities of $X$ we obtain a Calabi-Yau threefold with invariants

$$
\chi=56, \quad h^{1,1}=28, \quad h^{2,1}=0 .
$$

The threefold $X$ is investigated in detail in [6].

2.15. Type $T_{16}, h^{1,1}=16$. Consider the complete intersection threefold $X \subset \mathbb{P}^{5}$ defined by the equations

$$
\begin{aligned}
x_{0}^{2}+x_{1}^{2}+x_{2}^{2}+x_{3}^{2} & =4 x_{4} x_{5}, \\
x_{4}^{4}+x_{5}^{4} & =2 x_{0} x_{1} x_{2} x_{3} .
\end{aligned}
$$

The singular locus of $X$ consists of 12 singularities with local equation $x^{2}+y^{2}+z^{4}+t^{4}=0$ and 32 ordinary nodes. There exist projective small resolutions of all singularities of $X$. The invariants of a small resolution $\tilde{X}$ of $X$ are

$$
\chi(\tilde{X})=32, \quad h^{2,1}(\tilde{X})=0, \quad h^{1,1}(\tilde{X})=16 .
$$

The threefold $\tilde{X}$ is investigated in detail in [6].

\section{Geometrical constructions}

In this section we investigate several geometrical constructions leading to correspondences between Calabi-Yau threefolds.

Consider the two double octics $V_{1}$ and $V_{2}$ from 2.3 and observe that the branch loci are projectively equivalent to respectively

$$
\begin{array}{ll}
V_{1}: & z t(x+y)(x-y)(x+z)(t+y)(t+z)(t+y+z)=0, \\
V_{2}: & x t(x+z)(x-z)(x+y)(t+y)(t+z)(t+y+z)=0 .
\end{array}
$$

The Cremona transformation

$$
(x, y, z, t) \mapsto(y z, x y, x z, x t),
$$

which is a birational involution of $\mathbb{P}^{3}$, transforms one of them to the other. Thus the two double octics are birationally equivalent, as announced in 2.3 ,

Consider a homogeneous polynomial $F(x, y, z, t)$ of degree 4 . The $8: 1$ map

$$
\begin{gathered}
\mathbb{P}^{4}(1,1,1,1,4) \longrightarrow \mathbb{P}^{4}(1,1,1,1,4), \\
(x: y: z: t: w) \mapsto\left(x^{2}: y^{2}: z^{2}: t^{2}: x y z t w\right)
\end{gathered}
$$

induces a correspondence between the two double octics given by

$$
w^{2}=F\left(x^{2}, y^{2}, z^{2}, t^{2}\right)
$$


and by

$$
w^{2}=x y z t F(x, y, z, t) .
$$

If $F$ is a product of linear polynomials then the first octic is a union of four quadric surfaces and the second octic is an arrangement of eight planes. If we take $F(x, y, z, t)=(x-y)(y-z)(z-t)(t-x)$ then $F\left(x^{2}, y^{2}, z^{2}, t^{2}\right)=(x-y)(x+y)(y-z)(y+z)(z-t)(z+t)(t-x)(t+x)$, and we obtain a correspondence

$$
T_{44} \stackrel{8: 1}{\longrightarrow} T_{70}^{1} .
$$

Note that a correspondence between these varieties was already constructed implicitly by Nygaard and van Geemen in 8 .

If we take $F(x, y, z, t)=(x+y+z-t)(x+y-z+t)(x-y+z+$ $t)(-x+y+z+t)$ then we obtain a correspondence

$$
T_{28} \stackrel{8: 1}{\longrightarrow} T_{44} \text {. }
$$

Now assume that the double octic $X$ is given by

$$
w^{2}=f_{1}(x, y, z, t) f_{2}(x, y, z, t) f_{3}(x, y, z, t) f_{4}(x, y, z, t)
$$

with quadratic homogeneous polynomials $f_{i}(x, y, z, t)$. There is an obvious $8: 1$ correspondence between $X$ and the intersection $Y$ of four quadrics in $\mathbb{P}^{7}$ given by

$$
\begin{aligned}
& u_{1}^{2}=f_{1}(x, y, z, t), \\
& u_{2}^{2}=f_{2}(x, y, z, t), \\
& u_{3}^{2}=f_{3}(x, y, z, t), \\
& u_{4}^{2}=f_{4}(x, y, z, t) .
\end{aligned}
$$

For example, this construction produces a correspondence

$$
T_{32} \stackrel{8: 1}{\longrightarrow} T_{28} \text {. }
$$

As a second example consider the following intersection $X$ of four quadrics in $\mathbb{P}^{7}$ :

$$
\begin{aligned}
& u_{1}^{2}=x^{2}-y^{2}, \\
& u_{2}^{2}=y^{2}-z^{2}, \\
& u_{3}^{2}=z^{2}-t^{2}, \\
& u_{4}^{2}=t^{2}-x^{2} .
\end{aligned}
$$

This example is listed in section 2 as type $T_{40}^{3}$. We find the correspondence

$$
T_{40}^{3} \stackrel{8: 1}{\longrightarrow} T_{44}
$$


Consider also the composed map $X \longrightarrow \mathbb{P}_{[1,1,1,1,4]}^{4}$ defined by

$$
\phi\left(x: y: z: t: u_{1}: u_{2}: u_{3}: u_{4}\right)=\left(x^{2}: y^{2}: z^{2}: t^{2}: x y z t u_{1} u_{2} u_{3} u_{4}\right)
$$

It induces a correspondence

$$
T_{40}^{3} \stackrel{64: 1}{\longrightarrow} T_{70}^{1}
$$

Now we will describe a Calabi-Yau resolution $\tilde{X}$ of the singularities of $X$ and compute its Euler and Hodge numbers. The threefold $X$ is an iterated double covering of $\mathbb{P}^{3}$. The branch divisors are pairs of planes $\left(P_{1}, P_{2}\right),\left(P_{3}, P_{4}\right),\left(P_{5}, P_{6}\right),\left(P_{7}, P_{8}\right)$, intersecting along the lines $l_{i}=P_{2 i-1} \cap P_{2 i}$. Singularities of $X$ correspond to the lines $l_{i}$. The lines $l_{i}$ intersect in four points $Q_{1}=l_{1} \cap l_{2}, Q_{2}=l_{2} \cap l_{3}, Q_{3}=l_{3} \cap l_{4}, Q_{4}=l_{1} \cap l_{4}$. We first blow up $\mathbb{P}^{3}$ in the points $Q_{i}$ and then the strict transforms of the lines $l_{i}$. After these blow-ups the singular locus consists of eight ordinary nodes at the points $(1: \pm 1: \pm 1: \pm 1) \in \mathbb{P}^{3}$. The intersection of $X$ with the hyperplanes $u_{1}=\sqrt{-1} u_{2}, u_{3}=\sqrt{-1} u_{4}, x=z$ is a surface which contains four of the nodes and is smooth in these points (for the four remaining nodes take $x=-z$ instead). Thus there exist projective small resolutions.

We first compute the Euler characteristic of the singular model $X$. The idea is to stratify $\mathbb{P}^{3}$ by the number of points in the fibers of the iterated double cover. The generic fiber with 16 elements corresponds to a point outside the planes.

Counting points we see that the Euler characteristic of the sum of planes is $8 \cdot 3-28 \cdot 2+8+3 \cdot 12=12$ (we count 8 planes, then subtract 28 lines and finally take into account 8 points on three planes and 12 on four planes).

The set of fibers with 16 elements is the complement of the planes, so its Euler characteristic is $4-12=-8$.

Fibers with eight elements correspond to the points on the eight planes outside the double lines and also to the lines $l_{i}$ but without multiple points. The Euler characteristic of the sum of lines on one plane is $7 \cdot 2-3 \cdot 1-2 \cdot 6=-1$, hence the Euler characteristic of the complement of the lines in one plane is $3+1=4$. The line $l_{1}$ contains two fourfold and two threefold points, the Euler characteristic of the complement of multiple points in $l_{1}$ is 1 . In total the Euler characteristic of the set of fibers with eight elements is $8 \cdot 4+4(2-4)=$ 24.

There are exactly eight points whose fiber consists of one element. The rest contains fibers with four elements, its Euler characteristic is 
$4-(-8)-24-8=-20$. Finally the Euler characteristic of $X$ is

$$
\chi(X)=16(-8)+8 \cdot 24-4 \cdot 20+8=-8 .
$$

Now we will study the effect of blowing up a fourfold point. A generic point of the exceptional divisor (outside the double lines) has a fiber with 16 elements, the points on the lines have a fiber with 8 elements. There are six double points: four of them have fibers with 4 elements and two with 8 elements. Altogether the iterated cover of the exceptional plane has Euler characteristic $16 \cdot 1-8 \cdot 2+4 \cdot 4=16$. On $X$ there are four points over a fourfold point so the effect of blowing up fourfold points is $4(16-4)=48$.

Counting in the same manner for the blow-up of the double lines we obtain that the set of fibers with 16 and 8 elements has Euler characteristic 0 , and there are four points with fiber with 4 elements. Thus the effect of blowing up the double lines is $4(4 \cdot 4-4 \cdot 2)=32$. The small resolution of the eight nodes increases the Euler characteristic by 8. Finally we compute

$$
\chi(\tilde{X})=-8+48+32+8=80 .
$$

Now it is possible to compute the Hodge numbers of $\tilde{X}$ with van Geemen's point counting method. This requires counting points on the reduction $\bmod p$ for a sufficiently large prime $p$ such that Frobenius acts by multiplication with $p$ on $H^{2}(\tilde{X})$. For details the reader is referred to [6]. We find

$$
h^{1,1}(\tilde{X})=40, \quad h^{2,1}(\tilde{X})=0,
$$

so $\tilde{X}$ is rigid.

\section{RATIONAL ELLIPTIC FIBRATIONS}

Consider an arrangement $D$ of eight planes given by an equation

$$
f_{1} \cdot \ldots \cdot f_{8}=0
$$

and assume that among the eight planes there are two disjoint quadruples intersecting in a point each. After renumbering the equations and changing coordinates we can assume that $f_{1}, \ldots, f_{4}$ depend only on $x, y, z$, whereas $f_{5}, \ldots, f_{8}$ depend only on $y, z, t$.

Let $S$ and $S^{\prime}$ be the double coverings of $\mathbb{P}^{2}$ branched along the corresponding sums of four lines. Then in appropriate affine coordinates (f.i., $z=1$ ) they can be written as follows:

$$
\begin{aligned}
S & =\left\{(x, y, u) \in \mathbb{C}^{3}: u^{2}=f_{1}(x, y, 1) \cdot \ldots \cdot f_{4}(x, y, 1)\right\} \\
S^{\prime} & =\left\{(y, t, v) \in \mathbb{C}^{3}: v^{2}=f_{5}(y, 1, t) \cdot \ldots \cdot f_{8}(y, 1, t)\right\}
\end{aligned}
$$


This exhibits (birationally) both surfaces as elliptic fibrations. Moreover, the map

$$
((x, y, u),(y, t, v)) \mapsto(x, y, t, u v)
$$

is a rational, generically $2: 1$ map from their fiber product to the double covering of $\mathbb{P}^{3}$ branched along the octic surface $D$.

We will study elliptic fibrations coming from the above construction. We can get the following sequences of singular fibers:

$$
\left(I_{2}, I_{2}, I_{4}, I_{4}\right), \quad\left(I_{2}, I_{2}, D_{6}^{*}\right), \quad\left(I_{2}, I_{2}, I_{2}, D_{4}^{*}\right), \quad\left(I_{2}, I_{2}, I_{2}, I_{2}, I_{4}\right) .
$$

The first two examples are unique whereas the other two change in a one parameter family. In the table we give examples of explicit equations for the branch locus of corresponding double quartic elliptic fibrations. We also include the types and coordinates of the singular fibers and the Picard number $\rho\left(E_{w}\right)$ of the generic fiber (which can

\begin{tabular}{|c|c|c|c|c|c|}
\hline \multirow[t]{2}{*}{$S_{1}$} & $I_{2}$ & $I_{2}$ & $I_{4}$ & $I_{4}$ & \multirow{2}{*}{$\begin{array}{c}x(x+t)(x+z)(x+z+t) \\
\rho\left(E_{w}\right)=1\end{array}$} \\
\hline & 1 & -1 & 0 & $\infty$ & \\
\hline \multirow[t]{2}{*}{$S_{2}$} & $I_{2}$ & $I_{2}$ & $I_{4}$ & $I_{4}$ & \multirow{2}{*}{$\begin{array}{c}x(x+z+t)(x+z-t)(x+2 z) \\
\rho\left(E_{w}\right)=1\end{array}$} \\
\hline & 0 & $\infty$ & -1 & 1 & \\
\hline \multirow[t]{2}{*}{$S_{3}$} & $I_{2}$ & $I_{2}$ & $I_{2}$ & $D_{4}^{*}$ & \multirow{2}{*}{$\begin{array}{c}x(x+t)(x+\lambda t)(x+z) \\
\rho\left(E_{w}\right)=2\end{array}$} \\
\hline & 0 & 1 & $\lambda$ & $\infty$ & \\
\hline \multirow[t]{2}{*}{$S_{4}$} & $I_{2}$ & $I_{2}$ & $I_{2}$ & $D_{4}^{*}$ & \multirow{2}{*}{$\begin{array}{c}t(x+\lambda z)(x+z)(x+\lambda t) \\
\rho\left(E_{w}\right)=2\end{array}$} \\
\hline & 0 & 1 & $\lambda$ & $\infty$ & \\
\hline \multirow[t]{2}{*}{$S_{5}$} & $I_{2}$ & $I_{2}$ & $D_{6}^{*}$ & & \multirow{2}{*}{$\begin{array}{c}x t(x+z)(x+t) \\
\rho\left(E_{w}\right)=1\end{array}$} \\
\hline & 1 & 0 & $\infty$ & & \\
\hline \multirow[t]{2}{*}{$S_{6}$} & $I_{2}$ & $I_{2}$ & $D_{6}^{*}$ & & \multirow{2}{*}{$\begin{array}{c}x z(x+z)(x+t) \\
\rho\left(E_{w}\right)=1\end{array}$} \\
\hline & 1 & $\infty$ & 0 & & \\
\hline \multirow[t]{2}{*}{$S_{7}$} & $I_{2}$ & $I_{2}$ & $I_{2}$ & $I_{2}$ & \multirow{2}{*}{$\begin{array}{c}x(x+t)(x+z-\lambda t)(x+z) \\
\rho\left(E_{w}\right)=2\end{array}$} \\
\hline & 0 & 1 & $\lambda$ & $\lambda+1 \quad \infty$ & \\
\hline
\end{tabular}
easily be computed using the Zariski lemma).

For any of the above constructions it is not difficult to write down also a Weierstrass equation.

Remark 4.1. The fibrations $S_{3}$ and $S_{4}$ have the same types of special fibers but the quadruples of lines they are given by are not projectively equivalent. However, they can be transported to each other by a Cremona transformation. 
4.1. Isogeny between $S_{1}$ and $S_{2}$. Consider the elliptic fibration $S_{1}$ with congruence group $\Gamma_{1}(4) \cap \Gamma(2)$. Computing the Euler and Hodge numbers of the fiber product of $S_{1}$ and $S_{2}$ we obtain that the surfaces are isogenous. For a fixed $t$ the fibers come from each other by doubling the lattice in one direction.

If we have any elliptic curve $E$ with equation of the type

$$
y^{2}=x^{3}+A x^{2}+B x,
$$

then fixing the point at infinity as zero for the group structure the point $e=(0,0)$ becomes a half period. Dividing $E$ by the map $E \ni$ $p \mapsto p+e \in E$ we obtain as the quotient the curve

$$
y^{2}=(x+A)\left(x^{2}-4 B\right),
$$

and the quotient is given explicitly by the map

$$
(x, y) \mapsto\left(x+\frac{B}{x}, y-\frac{B}{x^{2}}\right) .
$$

We will apply this to the elliptic fibrations $S_{1}$ and $S_{2}$. The surface $S_{1}$ can be given in local coordinates by the (birationally equivalent) Weierstrass equation

$$
y^{2}=x\left(x-\left(t^{2}-1\right)\right)\left(x-t^{2}\right) .
$$

Let $S_{2}$ be the twist of $S_{1}$ by the automorphism of $\mathbb{P}^{1}$ given by $t \mapsto \frac{t-1}{t+1}$. Similar equations for $S_{2}$ have the form

$$
y^{2}=x\left(x-(t-1)^{2}\right)\left(x-(t+1)^{2}\right)
$$

Starting with (11) and replacing $x$ by $\frac{x}{4}$ and $y$ by $\frac{y}{8}$ we obtain

$$
y^{2}=x\left(x-4\left(t^{2}-1\right)\right)\left(x-4 t^{2}\right) .
$$

Replacing $x$ by $x+2\left(t^{2}-1\right)$ we get

$$
y^{2}=\left(x+2\left(t^{2}-1\right)\right)\left(x-2\left(t^{2}-1\right)\right)\left(x-2\left(t^{2}+1\right)\right) .
$$

Now replacing $x$ by $x+\frac{\left(t^{2}-1\right)^{2}}{x}$ and $y$ by $y\left(1-\frac{\left(t^{2}-1\right)^{2}}{x^{2}}\right)$ we obtain (2).

Composing the maps we find that the map $\phi: S_{2} \longrightarrow S_{1}$ given by

$$
\begin{aligned}
x & \mapsto \frac{x^{2}+16 x\left(t^{2}-1\right)+80\left(t^{2}-1\right)^{2}}{4\left(x+8\left(t^{2}-1\right)\right)} \\
y & \mapsto \frac{y}{8}\left(\frac{x^{2}+16 x\left(t^{2}-1\right)+48\left(t^{2}-1\right)}{\left(x+8\left(t^{2}-1\right)\right)^{2}}\right)
\end{aligned}
$$

is a generically $2: 1$ rational isogeny. 
4.2. Isogeny between $S_{1}$ and $X_{1128}$. Consider again the Weierstrass equation (11)

$$
\begin{aligned}
y^{2} & =x\left(x-\left(t^{2}-1\right)\right)\left(x-t^{2}\right) \\
& =x^{3}+\left(1-2 t^{2}\right) x^{2}+t^{2}\left(t^{2}-1\right) x
\end{aligned}
$$

for the fibration $S_{1}$. Proceeding as in 4.1, i.e., setting

$$
(x, y) \mapsto\left(x+\frac{t^{2}\left(t^{2}-1\right)}{x}, y-\frac{t^{2}\left(t^{2}-1\right)}{x^{2}}\right),
$$

we obtain the extremal fibration given by

$$
y^{2}=\left(x+1-2 t^{2}\right)\left(x^{2}-4 t^{2}\left(t^{2}-1\right)\right)
$$

with singular fibers of type $I_{1}, I_{1}, I_{2}, I_{8}$. In [7] this fibration is denoted by $X_{1128}$. The corresponding congruence group is $\Gamma_{0}(8) \cap \Gamma_{1}(4)$.

Thus there is a generically $2: 1$ rational map $\gamma: X_{1128} \longrightarrow S_{1}$.

4.3. Pullback from $S_{5}$ to $S_{1}$. The fibrations $S_{1} \cong S_{2}$ and $S_{5} \cong S_{6}$ are extremal, in [7] they are denoted by $X_{4422}$ and $X_{222}$. By [7] the fibration $S_{1}$ can be obtained from $S_{5}$ by a base change. To see this we substitute $t=t^{2}$ in the equation of $S_{5}$ in the above table and we obtain an equation of the form

$$
u^{2}=t^{2} x(x+1)\left(x+t^{2}\right) .
$$

Substituting $x=x-t^{2}$ and taking a normalization we get equation (1).

Denote by $\psi$ the map $\psi: S_{1} \longrightarrow S_{5}$. It is a generically $2: 1$ rational map. There is also a similar map $\psi^{\prime}: S_{1} \longrightarrow S_{6}$.

\section{Rigid double octic Calabi-Yau threefolds}

In this section we list the fiber products of elliptic fibrations which come from rigid double octic Calabi-Yau threefolds constructed from arrangements of eight planes and listed in [6] (we will also use the notations introduced there). Note that not all the elliptic fibrations are semistable, but they all admit crepant resolutions of singularities. We only have to consider the case of a product of $I_{n}$ and $D_{m}^{*}$. Since $D_{m}^{*}$ contains double lines the fiber product is singular along the product of such a line and a node. After blowing up all double lines there will remain only nodes which admit projective small resolutions.

The following table lists the double octics $X$ and their classification numbers (as in the tables in [6]) and invariants and a description of the corresponding (resolved) fiber products $Y$, including the types of the singular fibers and the Euler and Hodge numbers. We also give the 
levels of the corresponding weight four newforms. If the level is equal to 8 then we add the types $T$, as introduced in section 2 .

\begin{tabular}{|c|c|c|c|c|c|c|c|c|c|}
\hline \multicolumn{4}{|c|}{ double octic $X$} & \multicolumn{5}{|c|}{ (resolved) fiber product $Y$} & \multirow[b]{2}{*}{$N$} \\
\hline No. & $e$ & $h^{11}$ & $T$ & singular fibers & $e$ & $h^{11}$ & $h^{12}$ & $T$ & \\
\hline 1 & 140 & 70 & $T_{70}^{1}$ & $\begin{array}{ccc}I_{2} & D_{6}^{*} & I_{2} \\
D_{6}^{*} & I_{2} & I_{2}\end{array}$ & 72 & 36 & 0 & $T_{36}$ & 8 \\
\hline 3 & 124 & 62 & & $\begin{array}{cccc}I_{4} & I_{4} & I_{2} & I_{2} \\
D_{6}^{*} & I_{2} & I_{2} & I_{0}\end{array}$ & 88 & 45 & 1 & & 32 \\
\hline 19 & 108 & 54 & & $\begin{array}{cccc}I_{2} & I_{2} & I_{4} & I_{4} \\
I_{0} & D_{6}^{*} & I_{2} & I_{2} \\
\end{array}$ & 64 & 33 & 1 & & 32 \\
\hline 32 & 100 & 50 & $T_{50}$ & $\begin{array}{cccc}I_{2} & I_{2} & I_{4} & I_{4} \\
I_{2} & I_{2} & D_{4}^{*} & I_{2}\end{array}$ & 80 & 40 & 0 & $T_{40}^{2}$ & 8 \\
\hline 69 & 100 & 50 & $T_{50}$ & $\begin{array}{llll}I_{2} & I_{2} & I_{4} & I_{4} \\
I_{2} & I_{2} & D_{4}^{*} & I_{2}\end{array}$ & 80 & 40 & 0 & $T_{40}^{2}$ & 8 \\
\hline 93 & 92 & 46 & $T_{46}$ & $\begin{array}{cccc}I_{4} & I_{4} & I_{2} & I_{2} \\
I_{2} & I_{2} & D_{4}^{*} & I_{2}\end{array}$ & 64 & 32 & 0 & $T_{32}^{2}$ & 8 \\
\hline 238 & 88 & 44 & $T_{44}$ & $\begin{array}{llll}I_{2} & I_{2} & I_{4} & I_{4} \\
I_{2} & I_{2} & I_{4} & I_{4}\end{array}$ & 80 & 40 & 0 & $T_{40}^{1}$ & 8 \\
\hline 239 & 80 & 40 & & $\begin{array}{ccccc}I_{2} & I_{2} & I_{4} & I_{4} & I_{0} \\
I_{0} & I_{4} & I_{2} & I_{4} & I_{2}\end{array}$ & 64 & 34 & 2 & & 12 \\
\hline 240 & 80 & 40 & & $\begin{array}{ccccc}I_{2} & I_{2} & I_{4} & I_{4} & I_{0} \\
I_{2} & I_{2} & I_{2} & I_{4} & I_{2}\end{array}$ & 64 & 33 & 1 & & 6 \\
\hline 241 & 80 & 40 & $T_{40}$ & $\begin{array}{cccc}I_{2} & I_{2} & I_{4} & I_{4} \\
I_{4} & I_{4} & I_{2} & I_{2} \\
\end{array}$ & 64 & 32 & 0 & $T_{32}^{1}$ & 8 \\
\hline 245 & 76 & 38 & & $\begin{array}{ccccc}I_{2} & I_{2} & I_{4} & I_{4} & I_{0} \\
I_{4} & I_{2} & I_{2} & I_{2} & I_{2}\end{array}$ & 64 & 33 & 1 & & 6 \\
\hline
\end{tabular}

Observe that the fiber products are rigid exactly for the cases where the level is equal to 8. In the other cases, using the methods of [12, 5 ] we can identify codimension two modular motives in $H^{3}(Y)$. We can then show the modularity of the remaining two-dimensional motive, either by counting points over finite fields or by identifying it by the correspondence with the modular rigid double octic. 
Note also that the two level 32 newforms associated with the second and the third example are different so these two Calabi-Yau threefolds can not be in correspondence.

\section{Correspondences for level 8 Rigid Calabi-Yau THREEFOLDS}

In this section we use the results of sections 4 and 5 to describe further correspondences between level 8 rigid Calabi-Yau threefolds. In the end we compile all known correspondences into a picture.

Taking the fiber product of the maps $\phi$ and $\mathrm{id}_{S_{1}}$ we obtain a degree two correspondence between the Calabi-Yau threefolds that are resolutions of the fiber products $S_{2} \times_{\mathbb{P}^{1}} S_{1}$ and $S_{1} \times_{\mathbb{P}^{1}} S_{1}$ and hence a degree two correspondence between the Calabi-Yau threefolds defined by arrangements no. 241 and 238, i.e., there are correspondences

$$
T_{32}^{1} \stackrel{2: 1}{\longrightarrow} T_{40}^{1}, \quad T_{40} \stackrel{4: 2}{\longrightarrow} T_{44} .
$$

Taking the fiber product of the maps $\psi$ and $\psi^{\prime}$ we obtain a generically 4: 1 map from $S_{1} \times_{\mathbb{P}^{1}} S_{1}$ to $S_{5} \times_{\mathbb{P}^{1}} S_{6}$ and hence a degree four correspondence between the Calabi-Yau threefolds defined by arrangements no. 238 and 1, i.e., there are correspondences

$$
T_{40}^{1} \stackrel{4: 1}{\longrightarrow} T_{36}, \quad T_{44} \stackrel{8: 2}{\longrightarrow} T_{70}^{1} .
$$

Taking the fiber product of the maps $\phi$ and $\operatorname{id}_{S_{3}}$ we obtain a generically $4: 1$ map from $S_{2} \times_{\mathbb{P}^{1}} S_{3}$ to $S_{1} \times_{\mathbb{P}^{1}} S_{3}$ and hence a degree two correspondence between the Calabi-Yau threefolds defined by arrangements No. 32 and 93, i.e., there are correspondences

$$
T_{40}^{2} \stackrel{2: 1}{\longrightarrow} T_{32}^{2}, \quad T_{50} \stackrel{4: 2}{\longrightarrow} T_{46} .
$$

Taking the self-fiber product of the map $\gamma$ we obtain a generically 4: 1 map from $X_{1128} \times_{\mathbb{P}^{1}} X_{1128}$ to $S_{1} \times_{\mathbb{P}^{1}} S_{1}$ and hence a correspondence

$$
T_{70} \stackrel{4: 1}{\longrightarrow} T_{40}^{1} .
$$

Finally consider the complete intersection $X$ from 2.11(type $T_{32}$ ) and the Fermi threefold $Z$ from 2.1 (type $T_{70}$ ). J. Stienstra constructed the 8: 1 rational map $X \longrightarrow Z$ given by

$$
x=\frac{y_{0}+x_{0}}{y_{0}-x_{0}}, \quad y=\frac{y_{1}+x_{1}}{y_{1}-x_{1}}, \quad z=\frac{y_{2}+x_{2}}{y_{2}-x_{2}}, \quad t=\frac{y_{3}+x_{3}}{y_{3}-x_{3}},
$$

i.e., there is a correspondence

$$
T_{32} \stackrel{8: 1}{\longrightarrow} T_{70} .
$$

The map can be found in [8, page 60] but there are some misprints. 
Remark 6.1. We do not know whether the Calabi-Yau threefolds $T_{40}$, $T_{40}^{1}, T_{40}^{2}, T_{40}^{3}$ (resp. $T_{32}, T_{32}^{1}, T_{32}^{2}$ and $T_{70}, T_{70}^{1}$ ) are birational, however we belive that it is the case. In general we expect that any two rigid Calabi-Yau threefolds with equal Hodge numbers and the same $L_{-}^{-}$ series are birationally equivalent.

The following picture contains known correspondences between level 8 rigid Calabi-Yau threefolds. To keep it concise we only included those correspondences induced by explicit maps (including degrees). Correspondences that were known before are marked with thicker lines.

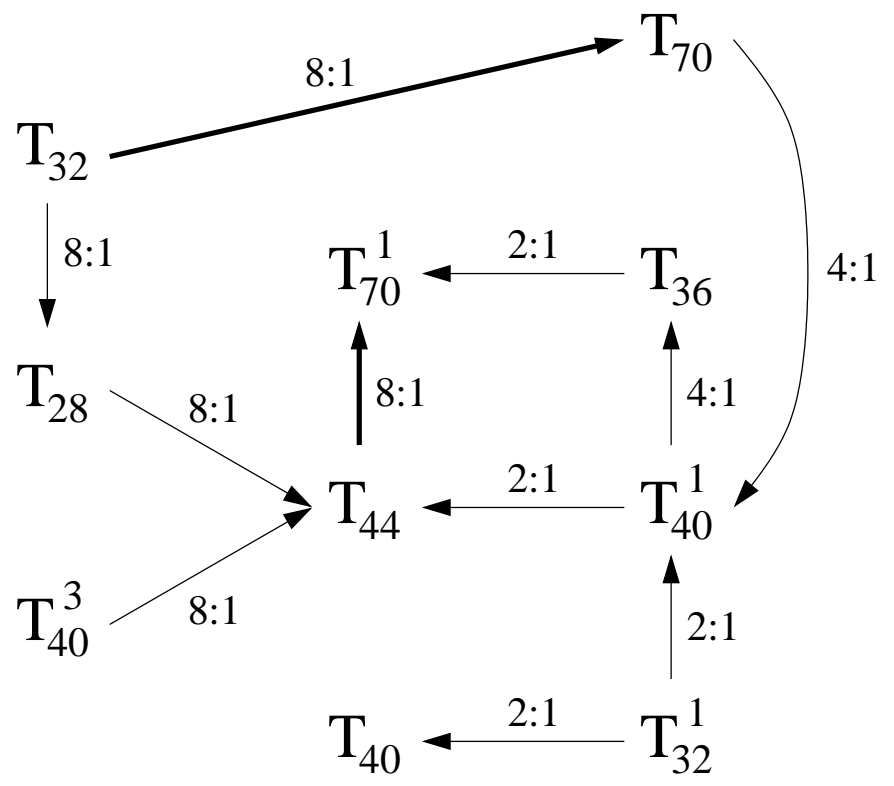

$\mathrm{T}_{16}$

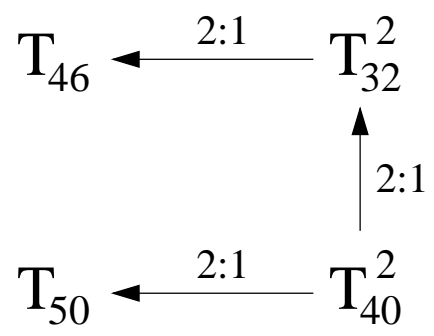

Note that there is a correspondence between $T_{16}$ and a double covering of $\mathbb{P}^{3}$ branched along the union of two Kummer surfaces with 12 common nodes. This correspondence is investigated in [6]. The Hodge numbers of the double octic have not yet been computed but numerical observations suggest that it is rigid. 


\section{Level 6 double octic Calabi-Yau threefolds}

In the table in section 5 there are two level 6 rigid double octic Calabi-Yau threefolds (constructed from arrangements no. 245 and 240).

Taking the fiber product of the maps $\phi$ and $\mathrm{id}_{S_{7}}$ we obtain a degree two correspondence between the Calabi-Yau threefolds that are resolutions of the fiber products $S_{2} \times_{\mathbb{P}^{1}} S_{7}$ and $S_{1} \times_{\mathbb{P}^{1}} S_{7}$ and hence a degree two correspondence between the two double octics.

Acknowledgements. The work on this paper was done during the first named author's stays at the Institutes of Mathematics of the Johannes Gutenberg-Universität Mainz and the Universität Hannover. He would like to thank both institutions for their hospitality. The authors also would like to thank Prof. Duco van Straten, Prof. Klaus Hulek and Matthias Schütt for their help.

\section{REFERENCES}

[1] V. Batyrev, Birational Calabi-Yau n-folds have equal Betti numbers, in Proceedings Warwick Euroconference 1996, eds. K. Hulek, F. Catanese, C. Peters, M. Reid, London Math. Soc. Lecture Note Ser. 264 (1999), Cambridge Univ. Press, pp. 1-11.

[2] S. Cynk, C. Meyer, Geometry and Arithmetic of Certain Double Octic CalabiYau Manifolds, to appear in Canadian Math. Bull.

[3] P. Deligne, Formes modulaires et reprsentations de GL(2), in Modular functions of one variable, II, Proc. Internat. Summer School, Univ. Antwerp, Antwerp (1972), pp. 55-105. Lect. Notes in Math. 349 (1973), Springer.

[4] K. Hulek, J. Spandaw, B. van Geemen, D. van Straten, The modularity of the Barth-Nieto quintic and its relatives, Adv. Geom. 1 (2001), pp. 263-289.

[5] K. Hulek, H. Verrill, On the modularity of Calabi-Yau threefolds containing elliptic ruled surfaces, preprint (2005), math.AG/0502158

[6] C. Meyer, A dictionary of modular threefolds, thesis, Mainz (2005).

[7] R. Miranda, U. Persson, On extremal rational elliptic surfaces, Math. Z. 193, no. 4 (1986), pp. 537-558.

[8] N. Nygaard, B. van Geemen, On the Geometry and Arithmetic of Some Siegel Modular Threefolds, J. Number Theory 53 (1995), pp. 45-87.

[9] C. Peters, J. Stienstra, A pencil of K3-surfaces related to Apéry's recurrence for $\zeta(3)$ and Fermi surfaces for potential zero, in Arithmetic of complex manifolds, Erlangen 1988, eds. W. Barth, H. Lange, Lect. Notes in Math. 1399 (1989), Springer, pp. 48-59.

[10] M. Saito, N. Yui, The modularity conjecture for rigid Calabi-Yau threefolds over $\mathbb{Q}$, J. of Math. Kyoto Univ. 41, no. 2 (2001), pp. 403-419.

[11] C. Schoen, On fiber products of rational elliptic surfaces with section, Math. Z. 197, no. 2 (1988), pp. 177-199.

[12] M. Schütt, On the modularity of three Calabi-Yau threefolds with bad reduction at 11, preprint (2004), math.AG/0405450 to appear in Canadian Math. Bull. 
[13] H. Verrill, The L-series of certain rigid Calabi-Yau threefolds, J. Number Theory 81 (2000), pp. 310-334.

[14] N. Yui, Update on the modularity of Calabi-Yau varieties, with appendix by H. Verrill, in Proceedings of the Workshop on "Calabi-Yau Varieties and Mirror Symmetry", Fields Institute, Toronto, July 23-29, 2001, eds. N. Yui, J. D. Lewis, Fields Inst. Comm. Series 38 (2003), AMS, pp. 307-362.

Instytut Matematyki, Uniwersytetu Jagiellońskiego, Ul. Reymonta 4, 30-059 Kraków, Poland

Current address: Institut für Mathematik, Universität Hannover, Welfengarten 1, D-30060 Hannover, Germany

E-mail address: s.cynk@im.uj.edu.pl

Institut für Mathematik, Johannes Gutenberg-Universität, StauDingerweg 9, D-55099 Mainz, Germany

E-mail address: cm@mathematik.uni-mainz.de 